\title{
Genetic Algorithm based Emotional State Evaluation from Filtered EEG Data
}

\author{
Charu Gitey \\ M. Tech. Scholar \\ Department of Computer Science \& Engineering \\ SIRT- Excellence \\ Bhopal, India \\ charugitey@gmail.com
}

\author{
Dr. Kamlesh Namdev \\ Associate Professor \\ Department of Computer Science \& Engineering \\ SIRT- Excellence \\ Bhopal, India \\ kamlesh.namdev@gmail.com
}

\begin{abstract}
Emotion plays an important role in the daily life of man and is an important feature of human interaction. Because of its role of adaptation, it motivates people to respond quickly to stimuli in their environment to improve communication, learning and decision making. With the increasing role of the braincomputer interface (BCI) in user-computer interaction, automatic recognition of emotions has become an area of interest in the last decade. The recognition of emotions could be facial expression, gesture, speech and text and could be recorded in different ways, such as electroencephalogram (EEG), positron emission tomography (PET), magnetic resonance imaging (MRI), etc. In this research work, feature extraction feature reduction and classification of emotions have been evaluated on different methods to recognize and classify different emotional states such as fear, sad, frustrated, happy, pleasant and satisfied from inner emotion EEG signals.
\end{abstract}

The aim of this research is to classify different emotions. The system's methodology consists of four main steps. In the first step signal processing is performed in which unnecessary artifacts are removed, for that butterworth filter is used to filter only required signals for analysis. In the second step DWT features are extracted and reduced using genetic algorithm. Further eight useful information from reduced DWT features are extracted. And in final step four classifier performance are analyzed for classification of the signals to the corresponding emotions. The proposed work gives highest accuracy with support vector classifier of $78.5 \%$ accuracy for arousal signal whereas for valence signal it gives approx. $78.9 \%$ accuracy.

Keywords:- EEG Signal, Emotion Recognition, EEG, BCI, Feature Extraction, Feature Reduction, Genetic Algorithm, Classification.

\section{INTRODUCTION}

Human emotion includes not only the psychological reaction of a human being to the external world or self-stimulation but also the physiological reaction to these psychological reactions. Human emotion is a combination of human thinking, feeling and behavior.

Emotion is a mental or physiological state that is subjective and private, which involves many feelings, thoughts, behaviors and actions. Emotions play a very important role in our daily lives. Emotion helps to make interaction with people fluid. A person's mood influences not only the way they interact with people, but also their actions. For example, feelings of anger or sadness can be fatal to both the driver and others. Emotions play a very important role in our daily activities. We tend to decide based on our moods. For example, if a man says "OK", he may say he is in a good mood or dissatisfied [1].

Therefore, it is important to study and recognize emotions. Emotions are easily captured by language, behavior and facial expressions [2]. But these can be falsely expressed by people. These pathways cannot be used effectively by patients with paralysis, strokes, ALS [3] or brain disorders. Therefore, the recognition of emotions is essential for people with conditions included. Therefore, it is important to study the physiological variables that vary with emotions [4].

Emotions represented along Valence Axis and Arousal axis results into four quadrants. Valence along $\mathrm{X}$-axis has been divided into two main classes called Positive Valence which means high and Negative Valence which means low and keeping the arousal state as constant [5]-[10]. 


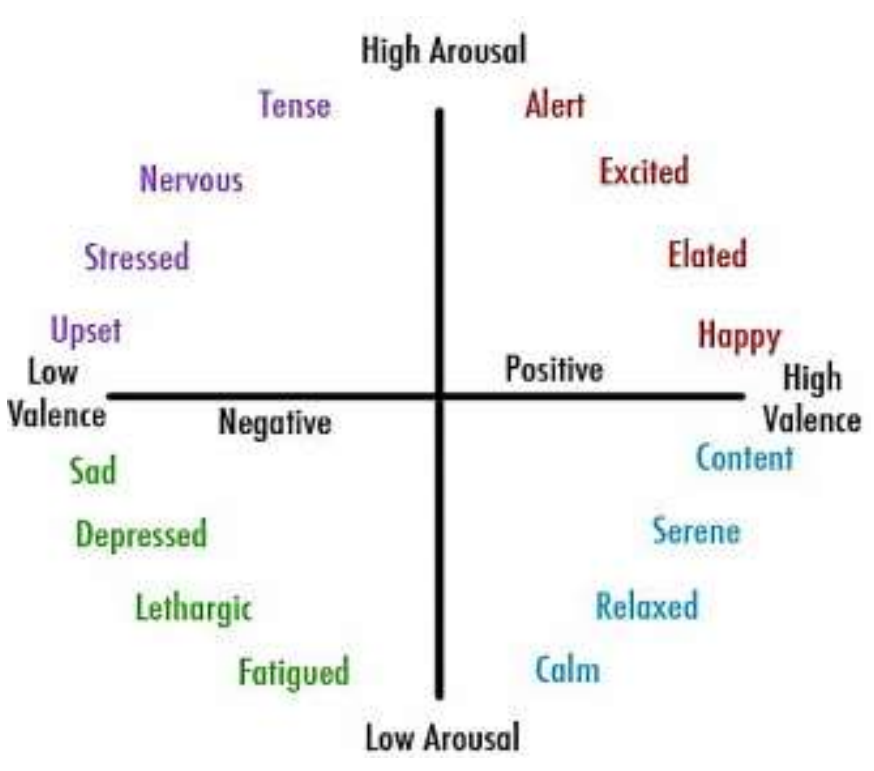

Figure 1: Dimension of Emotions

Likewise, arousal along Y- axis has been divided into two major classes namely Positive Arousal meaning high and Negative Arousal meaning low keeping the valence state as constant. It results into four major classes of emotions such as High Valence High Arousal (HVHA), Low Valence High Arousal (LVHA), High Valence Low Arousal (HVLA) and Low Valence Low Arousal (LVLA) [11]-[15]. Figure 1 shows the four classes of emotions.

\section{RELATED WORK}

Table 1 depicts the comparison based on above mentioned parameters. Feature extraction method indicates method used to extract the appropriate features from recorded EEG data related to emotions. Classification method indicates classifier used for discriminating different emotions, number of channels or electrodes of EEG recording device, frequency bands used for analyzing particular emotion, location of electrodes on brain. Time constraint specifies whether it is real time emotion recognition or not. All whole emotion recognition process is classified as good or better based on its percentage accuracy.

Table 1: Comparison of Existing EEG based Emotion Recognition

\begin{tabular}{|c|c|c|c|}
\hline Ref. & $\begin{array}{c}\text { Dataset and } \\
\text { Features }\end{array}$ & Technique & Accuracy \\
\hline$[15]$ & $\begin{array}{c}\text { Discrete Wavelet } \\
\text { Transform (DWT) }\end{array}$ & ANN & $55.58 \%$ \\
\hline
\end{tabular}

\begin{tabular}{|c|c|c|c|}
\hline [16] & $\begin{array}{l}\text { Raw data band } \\
\text { power feature of } 4 \\
\text { frequency bands }\end{array}$ & SVM & $65 \%$ \\
\hline [17] & $\begin{array}{l}\text { Raw data and } \\
\text { individual } \\
\text { normalization } \\
\text { Entropy and } \\
\text { Energy of gamma } \\
\text { frequency band }\end{array}$ & KNN & $\begin{array}{l}86.75 \% \\
\text { Valence } \\
84.05 \% \\
\text { Arousal }\end{array}$ \\
\hline [18] & $\begin{array}{l}\text { Preprocessed data } \\
\text { statistical features, } \\
\text { band power, Hjorth } \\
\text { parameters and } \\
\text { fractal dimension }\end{array}$ & SVM & $\begin{array}{l}73.14 \% \\
\text { Valence } \\
73.06 \% \\
\text { Arousal }\end{array}$ \\
\hline [19] & $\begin{array}{l}\text { Preprocessed data } \\
\text { power ratio, power } \\
\text { spectral density, } \\
\text { entropy, Hjorth } \\
\text { parameters and } \\
\text { correlation }\end{array}$ & ANN & $\begin{array}{l}72.87 \% \\
\text { Valence } \\
75.00 \% \\
\text { Arousal }\end{array}$ \\
\hline [20] & $\begin{array}{l}\text { Preprocessed data } \\
\text { PSD and DE }\end{array}$ & SVM & $\begin{array}{l}85.20 \% \\
\text { Valence } \\
80.50 \% \\
\text { Arousal }\end{array}$ \\
\hline [21] & $\begin{array}{l}\text { Preprocessed data } \\
\text { and channel } \\
\text { normalization } \\
\text { Entropy and } \\
\text { Energy of gamma } \\
\text { frequency band }\end{array}$ & $\begin{array}{l}\text { KNN (10- } \\
\text { fold cross- } \\
\text { validation } \\
\text { all samples) }\end{array}$ & $90 \%$ \\
\hline
\end{tabular}

The use of BCI emotion recognition systems presents several challenges such as [22]-[23]:

- Noise removal is also an important issues.

- Important features are required for proper classification of emotions.

For example, choose the method and channels to acquire brain signals that provide the best possible information about a person's emotional state and classifiers to achieve good accuracy in recognizing emotions [24]-[26].

\section{PROPOSED METHODOLOGY}

The aim of this research is to classify different emotions as illustrated in figure 2. The system's methodology consists of 
four main steps. The first step was Signal acquisition. The second step was signal preprocessing, to remove the noises and unwanted data. The third step was features extraction from the EEG signals. The fourth step was classification of the signals to the corresponding emotions.

The performance of proposed algorithm is illustrated by performing the steps as mentioned in the figure 2 and their discussions are given below:

\section{A. Signal Acquisition}

The recording comes from [26], a registered subject for the EEG data taken by the participants. The greater the number of participants, the better the results. We had student participants. The total number of participants was 34, including 20 men and 14 women (average age: 20.5 years). Most of the EEG data records for one day with total time for each participant was 25 minutes.

Emotiv EPOC is a device used to obtain EEG data from the participants. As shown in Figure 2, there are 14 channels used in Emotiv EPOC. Their channel names are AF3, AF4, F3, F4, F7, F8, FC5, FC6, P7, P8, T7, T8, O1 and O2. The sampling frequency used in the device is $128 \mathrm{~Hz}$.

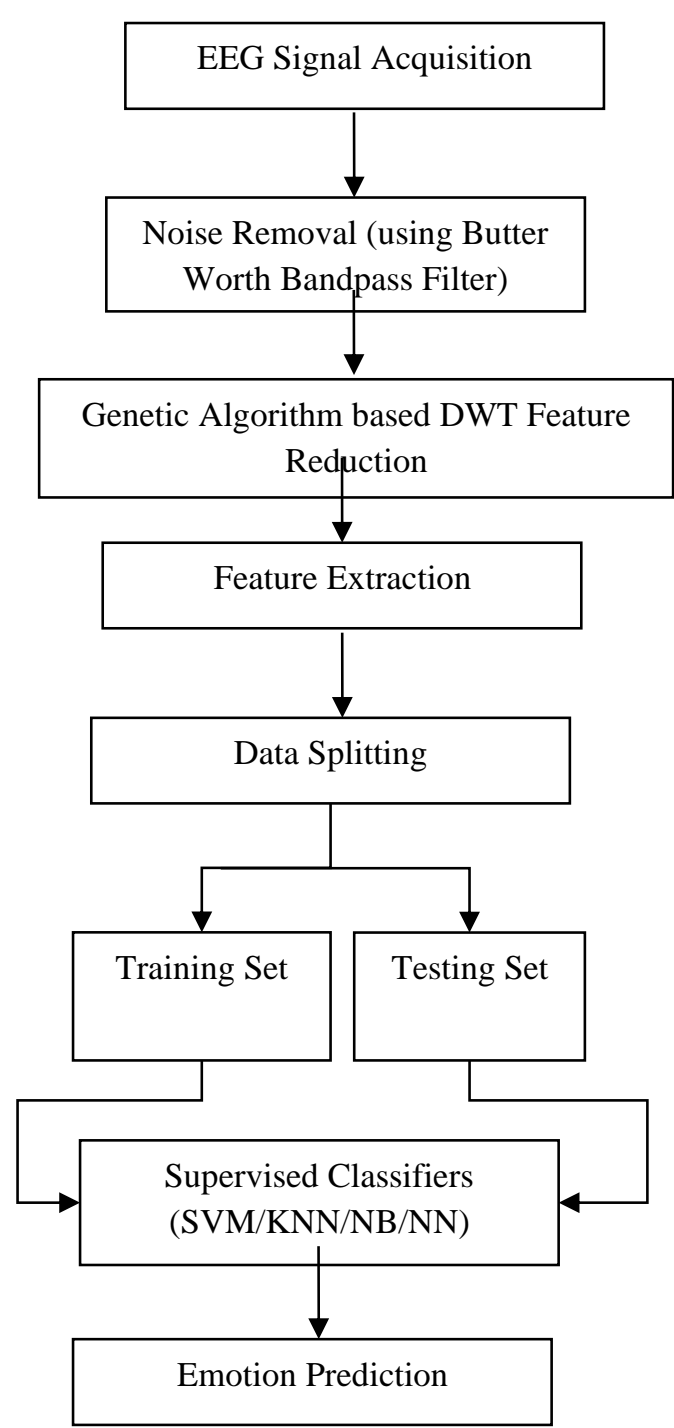

Figure 2: Flow Chart of Proposed Methodology

\section{B. Preprocessing}

The EEG signals were filtered with band pass filter using Butterworth filter to remove the unwanted artifacts. Using a Band pass filter to reduce the frequency band used, reducing the numbers of channels (channel selection) and reducing the number of features, have a direct impact on reducing the execution time, and increasing the utilization of memory which enhance the system performance.

\section{Feature Extraction}

Features extraction from the EEG signals were implemented using different techniques to compare. Since the EEG is a nonstationary signal many methods such as time domain, frequency domain, and time-frequency domain methods were used. Discrete Wavelet transform (DWT) was the most 
appropriate choice to use time-frequency domain methods as a vital for feature extraction [13].

Discrete Wavelet Transform (DWT) is used for feature extraction for emotion signals and further reduced these DWT features using genetic algorithm and further these reduced DWT features are used to find further eight values like mean, median, variance, standard deviation, skewness, kurtosis, complexity and mobility for classifying the extracted features.

\section{Dimension Reduction and Feature Selection}

After extraction some appropriate signals' features, a feature vector for each trials $\mathrm{Y}=[\mathrm{y} 1, \mathrm{y} 2 \ldots \mathrm{yn}]$ is achieved. Each feature vector's dimension for feed to the classifiers should be reduced, because the classifiers suffers from the curse of dimensionality. Having a matrix, A with size $\mathrm{n} \times \mathrm{m}$ in the way that the number of its column (m) is smaller than its number of rows (n), by reducing the size of the matrix $\mathrm{A}$, a matrix $\mathrm{Z}$ can be derived which its dimension in the column (m) significantly smaller than the original matrix. By linear transformation $\mathrm{Z}=$ $\mathrm{A}^{\mathrm{T}} \mathrm{Y}$ and an initial vector $\mathrm{Y}$, the original matrix A projected into the vector $\mathrm{Z}$ which it can be reduced to lower dimension.

Transformation in this way, makes some information in the original matrix will be lost whereas classification can be easier. Generally, there is a tradeoff between amount of losing information and classification simplicity level which must be considered. There are several methods in the studies which can reduce the dimension of feature vectors such as genetic algorithm (GA).

Algorithm of genetic algorithm are discussed below and shown in figure 4.2 :

Procedure: GENETIC ALGORITHM (P)

$\mathrm{t} \leftarrow 0$

InitPopulationP $(\mathrm{t}) \rightarrow$ Initialize Population randomly

$\mathrm{F}(\mathrm{t})=$ ComputeFitness $(\mathrm{P}(\mathrm{t}))$

while not terminated do

$\mathrm{t} \leftarrow \mathrm{t}+1$

$\mathrm{P}(\mathrm{t}) \leftarrow \operatorname{crossover}(\mathrm{P}(\mathrm{t}-1))$

$\mathrm{P}(\mathrm{t}) \leftarrow \operatorname{mutate}(\mathrm{P}(\mathrm{t}))$

$\mathrm{F}(\mathrm{t}) \leftarrow$ ComputeFitness $(\mathrm{P}(\mathrm{t}))$

end while

return the best individual

end procedure
After DWT feature reduction using genetic algorithm some useful information are extracted that represent the characteristics of original signal without redundancy. These are discussed below:

\section{i. Mean}

Mean relates to the focus/center of a set of value. The Mean is considered for each and every sub-band signals [14].

$$
\text { Mean }=1 / N \sum_{i=1}^{n} X i
$$

\section{ii. Median}

The median [14] is the value splitting the upper half of a data sample/a population, or a probability distribution, from the lower half. In modest relations, it may be believed of as the "intermediate" value of a data set.

iii. Variance

The average of the squared differences from the Mean.

$$
\sigma=(x+a)^{n}=\sum_{i=1}^{n}(X-\mu)^{2} / N
$$

\section{iv. Standard deviation}

Standard deviation [15] is a simple measure of the changeability of a data set. The Standard deviation is the rootmean-square (RMS) deviation of its values from the mean.

$$
\operatorname{std}=\sqrt{\left.\left(\sum_{\mathrm{i}=1}^{\mathrm{n}}(\mathrm{Xi}-\mathrm{X})^{2}\right)\right) / \mathrm{N}-1}
$$

\section{vi. skewness}

Skewness is a degree of the asymmetry of the probability distribution of a real-valued random variable about its mean[15,16]. The skewness value can be either positive or negative, or even undefined.

$$
\text { skewness }=\mathrm{E}\left[(\mathrm{X}-\mu \sigma)^{3}\right.
$$

\section{vii. Kurtosis}

Kurtosis[15] characterizes the comparative peakedness or flatness of a distribution associated with the normal distribution. This is given by: 


$$
\text { kurtosis }=\mu 4 / \sigma^{4}
$$

where, $\mu 4$ is the fourth moment about the mean and $\sigma$ is the standard deviation.

\section{ix. Complexity}

The Complexity parameter [15] signifies the variation in frequency.

$$
\text { Complexity }=\operatorname{Mobility}\left(\frac{\mathrm{dy}(\mathrm{t})}{\mathrm{dt}}\right) / \operatorname{Mobility}(\mathrm{y}(\mathrm{t}))
$$

\section{x. Mobility}

The mobility $[15,16]$ parameter signifies the mean frequency or the proportion of standard deviation of the power spectrum.

$$
\text { Mobility }=\sqrt{\operatorname{var}\left(\frac{\mathrm{dy}(\mathrm{t})}{\mathrm{dt}}\right) / \operatorname{var}(\mathrm{y}(\mathrm{t}))}
$$

\section{E. Feature Classification}

When desired features have been extracted, it still need to teach the system to recognize the user's emotion from brain signal, which it can be accomplished by a classifier. There are several method of classifier which used in the classification of EEG signals such as Support Vector Machine (SVM), Artificial Neural Network (NN), K Nearest Neighbour (k-NN) and Naïve Bayes (NB).

\section{Proposed Algorithm}

Input: $\mathrm{D}\{\mathrm{EEG}$ data $\}$;

Output: Label $\{$ Emotion Label\};

Emotion's Label\{Normal, Emotion\}

Step1: Pre-processing and data cleansing

Step2: For each instance in D, do

Find DWT feature vector (V)

Step 3: For each V do

Data reduction using Genetic Algorithm and split data in two halves and classify data using SVM, KNN, NN and NB algorithm

Step 4: Determine the total class label

Find

True_positive (TP)
True_negative (TN)

False_positive (FP)

False_negative (FN)

Step 5: Find Performance Parameters

Step 6: Predict Emotion Class as stated below rules:

The proposed model categorizes human emotions into six classes based on valence and arousal as described below:

1) Class 1, High Arousal

2) Class 2, Low Arousal

3) Class 3, Normal Arousal

4) Class 4, High Valence

5) Class 5, Low Valence

6) Class 6, Normal Valence

Further for deciding the emotion following rules are followed as:

Rule 1: High Arousal Low Valence (HALV) representing anger, upset, stress, and frustrated.

Rule 2: High Arousal High Valence (HAHV) representing happy, excited, and interested.

Rule 3: Low Arousal High Valence (LAHV) representing relief, relaxed, and comfortable.

Rule 4: Low Arousal Low Valence (LALV) representing tired, bored, and sad.

end for

\section{RESULT ANALYSIS}

A. Classifier Comparison using Genetic Algorithm

In this section, the proposed methodology is performed and evaluated the accuracy of the arousal and valence of the system. For that, the proposed methodology is compared with four different classifier i.e. support vector machine, k-nearest neighbor, neural network and naïve bayes. Form result it is seen that support vector machine provides an accuracy of about $78 \%$ for both arousal and valence as compared to other. Table 2 represents the performance of all classifiers for proposed scheme. 
Table 2: Performance Analysis of Proposed Methodology

\begin{tabular}{|l|r|r|r|}
\hline Classifiers & \multicolumn{1}{|c|}{$\begin{array}{c}\text { Arousal } \\
\text { Accuracy }\end{array}$} & \multicolumn{1}{|c|}{$\begin{array}{c}\text { Valence } \\
\text { Accuracy }\end{array}$} & $\begin{array}{c}\text { Overall } \\
\text { Accuracy }\end{array}$ \\
\hline GA-SVM & $78.59 \%$ & $78.96 \%$ & $78.77 \%$ \\
\hline GA-KNN & $71.73 \%$ & $69.57 \%$ & $70.65 \%$ \\
\hline GA-NB & $63.56 \%$ & $57.44 \%$ & $60.50 \%$ \\
\hline GA-NN & $65.84 \%$ & $67.63 \%$ & $67.72 \%$ \\
\hline
\end{tabular}

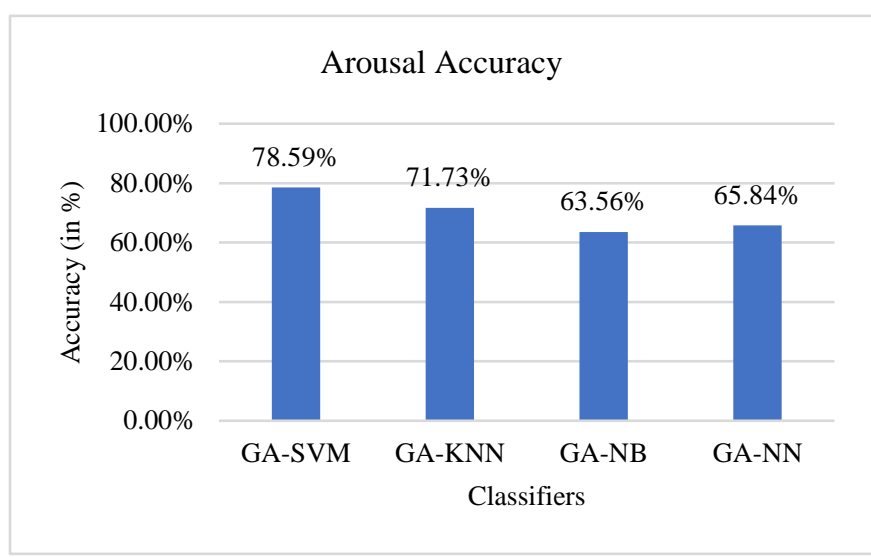

Figure 3: Arousal Performance Analysis

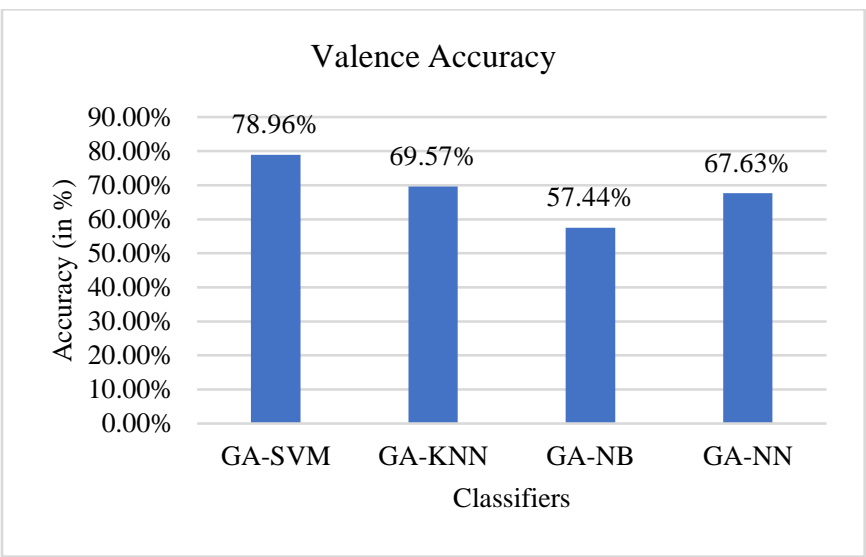

Figure 4: Valence Performance Analysis

Figure 3 represents the arousal accuracy and shows that support vector machine achieves highest accuracy and naïve bayes achieved lowest accuracy. Whereas figure 4 represents the valence accuracy performance and it has been observed that in this also support vector machine achieves highest accuracy and naïve bayes achieves lowest accuracy. So, the overall accuracy of the support vector machine is highest as compared to all three classifiers.

\section{B. Comparison with Existing Work}

In existing work [1], feature extraction and classification of emotions have been evaluated on different methods to recognize and classify six emotional states such as fear, sad, frustrated, happy, pleasant and satisfied from inner emotion EEG signals. The results showed that using appropriate feature for extraction emotional state such as Discrete Wavelet Transform (DWT) and Artificial Neural Network (ANN) and achieved an accuracy of $55.58 \%$. In existing technique [1] there is no noise removal technique is used as well as discrete wavelength transform is used for feature extraction and then PCA feature reduction technique is applied with neural network, SVM, NB and KNN classifiers.

After analyzing the result, it is concluded that proposed algorithm based on genetic algorithm based reduced DWT features with SVM classifier outperforms better as compared to other techniques.

Table 3: Comparative Performance Analysis with Existing Work

\begin{tabular}{|c|r|c|}
\hline Classifiers & $\begin{array}{c}\text { Genetic } \\
\text { Algorithm } \\
\text { (Proposed) }\end{array}$ & $\begin{array}{c}\text { PCA } \\
\text { (Existing) [1] }\end{array}$ \\
\hline SVM & $78.77 \%$ & $55.58 \%$ \\
\hline KNN & $70.65 \%$ & $51.82 \%$ \\
\hline NB & $60.50 \%$ & $49.82 \%$ \\
\hline NN & $67.72 \%$ & $50.70 \%$ \\
\hline
\end{tabular}

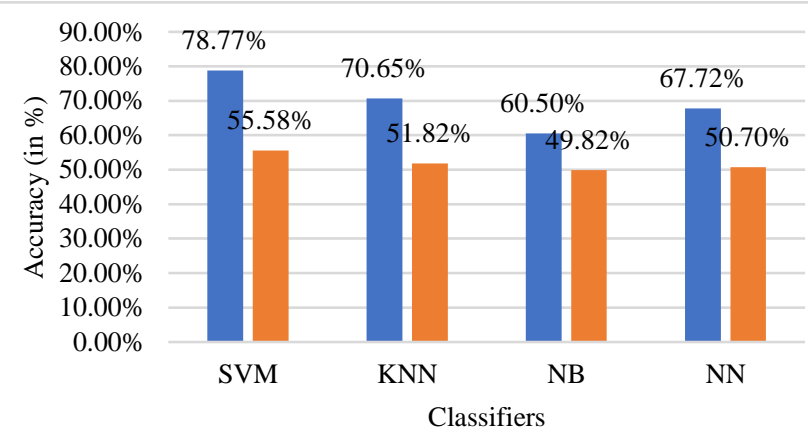

- Genetic Algorithm (Proposed) $\quad$ PCA (Existing) [1]

Figure 5: Comparative Performance Analysis with Existing Work

Table 3 and figure 5 shows the overall accuracy of the proposed model and existing model and describes that proposed model is about $30 \%$ more efficient as compared to existing work.

\section{CONCLUSION}

The recognition of emotions is very important, especially in terms of application, for example patient monitoring and the treatment management system of this patient. In this work, an EEG-based emotion recognition system is developed, 
consisting of a feature extraction subsystem and a classification subsystem. This research presents the development of an emotion recognition system based on the processing of the EEG signal.

This research presents the development of an emotion recognition system based on the processing of the EEG signal of different emotional states. The proposed work is used to design and implement for genetic algorithm based DWT feature reduction and classification of different emotional states using supervised artificial intelligence approaches such as SVM, KNN, NB and NN. It has been observed that the genetic algorithm optimized DWT features gives better accuracy as compared to existing PCA technique. The proposed work gives highest accuracy with support vector classifier of $78.5 \%$ accuracy for arousal signal whereas for valence signal it gives approx. $78.9 \%$ accuracy. This research work gives about $30 \%$ improved accuracy with the existing technique.

\section{REFERENCES}

[1] Mostafa Mohammadpour, Seyyed Mohammad Reza Hashemi, Negin Houshmand, "Classification of EEG-based Emotion for BCI Applications", IEEE, 2017.

[2] Y.P. Lin, C.H. Wang, T.P. Jung, T.L. Wu, S.K. Jeng, J.R. Duann, and J.H. Chen, "EEG-Based Emotion Recognition in Music Listening," IEEE Transactions on Biomedical Engineering IEEE Trans. Biomed. Eng., Vol. 57, No. 7, pp. 1798-1806, 2010.

[3] A.Choppin, "EEG-based human interface for disabled individuals: Emotion expression with neural networks," Master's thesis, Tokyo Institute of Technology, 2000.

[4] E. Niedermeyer, and F.H. Lopes da Silva, "Electroencephalography: Basic Principles, Clinical Applications, and Related Fields," Lippincot Williams \& Wilkins, 2005.

[5] M. Othman, A. Wahab, and R. Khosrowabadi, "MFCC for robust emotion detection using EEG," in In proceedings of the 2009 IEEE 9th Malaysia International Conference on Communications, December 2009.

[6] R.J. Wolpaw, N. Birbaumer, D. J. McFarland, G. Pfurtscheller, and T. M. Vaughan, " Brain- computer interfaces for communication and control,”Clinical Neurophysiology, Vol. 113, No. 6, pp. 767-791, March 2002.

[7] J. Wang, , N. Yan, H. Liu, M. Liu, and C. Tai, “ Brain-computer interfaces based on attention and complex mental tasks," In Proceedings of International Conference on Digital Human Modeling, pp. 467-473, Springer Berlin Heidelberg, 2007.

[8] R.Plutchik, "The Emotions: Facts, Theories and a New Model," Random House, New York, 1962.

[9] P. Ekman, W. V. Friesen, M. O'Sullivan, A. Chan, I. D.-Tarlatzis, K. Heider, and R. Krause, "Universals and Cultural Differences in the Judgments of Facial Expressions of Emotion," Journal of Personality and Social Psychology, Vol. 53, No. 4, pp. 712-717, October 1987.

[10] J.A.Russell, "A Circumflex Model of Affect," Journal of Personality and Social Psychology, Vol. 39, pp. 1161-1178, 1980.

[11] M. M. Bradley, and P. J. Lang, "Measuring emotions: The self assement manikin and the semantic differential," Journal of behavior therapy and experimental psychiarty, Vol. 25, No.1, pp. 49-59, March 1994.

[12] Van Erp J, Lotte F, Tangermann M. "Brain-computer interfaces: beyond medical applications" IEEE Computer, 2012, Vol. 45, Issue 4, pp 26-34.
[13] Bi L, Fan X-A, Liu Y., "EEG-based brain-controlled mobile robots: a survey", Human-Machine Syst, IEEE Trans, 2013, Vol. 43, Issue 2, pp. 161-76.

[14] L. F. Nicolas-Alonso and J. Gomez-Gil, "Brain computer interfaces, a review," Sensors, 2012, Vol. 12, Issue. 2, pp. 1211-1279.

[15] D. Göhring, D. Latotzky, M. Wang, and R. Rojas, "Semi-autonomous car control using brain computer interfaces," Intelligent Autonomous Systems, Springer, 2013, Vol. 194, pp. 393-408.

[16] D. J. McFarland, C. W. Anderson, K. Muller, A. Schlog1, and D. J. Krusienski, "Bci meeting 2005-workshop on bci signal processing: feature extraction and translation," IEEE transactions on neural systems and rehabilitation engineering, 2006, Vol. 14, Issue. 2, pp. 135.

[17] M. M. Moore, "Real-World Applications For Brain-Computer Interface Technology," Neural Systems and Rehabilitation Engineering, IEEE Transactions, 2003, Vol.11, Issue. 2, pp. 162-165.

[18] P. W. Ferrez\& J. del R Millan, "Error-related eeg potentials generated during simulated Brain computer interaction," Biomedical Engineering, IEEE Transactions, 2008, Vol 55, Issue. 3, pp. 923-929.

[19] C.A. Frantzidis, C. Bratsas, C.L. Papadelis, E. Konstantinidis, C. Pappas, P.D. Bamidis," "Toward emotion aware computing: an integrated approach using multichannel neuro-physiological recordings and affective visual stimuli", IEEE Transaction on Information Technology and Biomedical, Vol. 14 pp. 589-597, 2010.

[20] E.I. Konstantinidis , C.A. Frantzidis , C. Pappas , P.D. Bamidis , "Real time emotion aware applications: a case study employing emotion evocative pictures and neuro-physiological sensing enhanced by graphic processor units", Computer Methods and Programs in Biomedicine. Vol 107, pp. 16-27, 2012.

[21] D. Iacoviello , A. Petracca , M. Spezialetti , G. Placidi , “A real-time classification al- gorithm for EEG-based BCI driven by self-induced emotions", Comput. Methods Prog. Biomed. Vol. 122, pp. 293-303, 2015 .

[22] M. Khezri , M. Firoozabadi, A.R. Sharafat "Reliable emotion recognition system based on dynamic adaptive fusion of forehead biopotentials and physiological signals", Comput. Methods Prog. Biomed. Vol. 122, pp. 149-164, 2015.

[23] G.K. Verma , U.S. Tiwary, "Multimodal fusion framework: a multiresolution approach for emotion classification and recognition from physiological signal", NeuroImage, Vol. 102, pp. 162-172, 2014.

[24] X. Li, P. Zhang, D. Song, G. Yu, Y. Hou, B. Hu, "EEG based emotion identification using unsupervised deep feature learning", SIGIR2015 Workshop on Neuro-Physiological Methods in IR Research, Vol. 13, 2015.

[25] X. Jia, K. Li , X. Li , A. Zhang, “A Novel semi-supervised deep learning framework for affective state recognition on EEG signals", IEEE 14th International Conference on Bioinformatics and Bioengineering, 2014.

[26] Noufal Sulthan, "Emotion Recognition Using Brain Signals", IEEE, 2018. 